\title{
1 Experimental Optimisation of Power for Large Arrays of Cross-Flow Tidal Turbines
}

\author{
3 Duncan Sutherland ${ }^{\mathrm{a}, *}$, Stephanie Ordonez-Sanchez ${ }^{\mathrm{b}}$, Michael R. Belmont ${ }^{\mathrm{c}}$, \\ ${ }_{4} \quad$ Ian Moon ${ }^{\mathrm{c}}$, Jeffrey Steynor ${ }^{\mathrm{d}}$, Thomas Davey ${ }^{\mathrm{d}}$, Tom Bruce $^{\mathrm{a}}$ \\ ${ }_{5}{ }^{a}$ Institue for Energy Systems, School of Engineering, University of Edinburgh \\ ${ }^{b}$ Energy Systems Research Unit, University of Strathclyde \\ ${ }^{c}$ College of Engineering, Mathematics and Physical Sciences, University of Exeter, \\ University of Exeter \\ ${ }^{d}$ FloWave Ocean Energy Research Facility, University of Edinburgh
}

Preprint shabland @ed aquk URL: www.eng.ed.ac.uk/research/institutes/ies (Duncan Sutherland) www.strath.ac.uk/esru/research/hydrowavetidalpower/ (Stephanie Ordonez-Sanchez), emps.exeter.ac.uk/engineering/research/cws (Michael R. Belmont), www.flowavett.co.uk/ (Thomas Davey)

The layouts investigated explore the effect of lateral and stream-wise turbine spacings as well as differences between staggered and in-line layouts on power. The staggered array with decreased streamwise spacing is shown to have the highest total power per 'footprint' area among the layouts tested. For the staggered arrays, increased downstream separation had little effect on total power generated, while decreasing the lateral spacing below 2 rotor diameters decreased the power. The in-line arrays showed a lower power per device but similar total power. It was also shown that increased in- 

$48 \quad[6,7,8,9]$.

flow into a turbine didn’t necessarily lead to an increased power extraction. The decrease in power with a decrease in streamwise spacing is in-line with theoretical and CFD predictions.

Keywords: Renewables, Tidal Energy, Arrays, Scale Testing, Wake Interactions, Physical Modelling

\section{Introduction}

Tidal energy is considered a potentially significant contributor to the UK's energy mix, with estimates ranging from $15.7 \mathrm{TWh} /$ year [1] and 20.6 TWh/year [2] which would account for $4.6 \%$ to $6.1 \%$ of the UKs electricity requirements [3]. With several commercial scale prototypes tested in isolation, the focus of the hydrodynamic research has shifted towards both second generation technologies optimised for specific environments and the interaction of devices in arrays. This work focuses on the novel Momentum Reversal Lift (MRL) turbine designed by the University of Exeter in conjunction with Aquascientific Ltd, using up to 15 scale models in a variety of array configurations to assess the effect of layout and spacing on power output. The optimum spacing for turbines is critical to extract maximum power and to predict loadings in arrays and is a field which has had limited experimental testing given the stage of commercial array projects. This work builds upon the work by: Janssen and Belmont [4] and Ordonez et al. [5] assessing the extractable power and wake evolution in both an individual turbine and a four turbine array, as well as CFD work on both device and array optimisation 


\section{Background}

\subsection{Tidal Turbine Arrays}

Flow through an array of turbines is highly complex, due to the nature of tidal energy sites $[10,11]$ and the interaction of turbine wakes $[12,13,14]$. Energy extraction devices in tidal channels can in theory utilise high global blockage ratios, i.e. the ratio of total turbine swept area divided by the channel cross-sectional area. By doing so they are in theory able to extract a greater percentage of available power than in an open channel, increasing the Lanchester-Betz ratio of 0.593 to 0.798 [15]. Staggering devices in rows use upstream turbines, which provide local blockage, to accelerate flow between them, so that the downstream turbines have a higher inflow velocity. The use of these arrays has been theorised to increase extractable power for certain downstream spacings [16]. To this end, several studies have focused on wake evolution and downstream mixing $[17,12]$ with the goal of maximisation of the local available power in the flow, which is expected to increase with the cube of the flow speed. In addition, increasing the downstream spacing between rows allows the wake after the first row to mix with the bypass and free-stream flow to recover to a higher value, increasing the inflow to the subsequent row. In principle it is possible that as a turbine causes bypass flow acceleration around it, the downstream flow can be higher than the upstream flow despite kinetic energy being extracted by the turbine, as the total energy is conserved through a loss of head.

Local inflow velocity is not the only factor that will effect the extractable power for a turbine. It is generally agreed that a more turbulent flow for the equivalent velocity will induce less lift which in turn will reduce the power 
that a turbine can extract. However, these effects are complex and depend upon the scales of turbulence in question $[18,11]$. Turbines are also sensitive to flow direction which changes the effective angle of attack of the lifting surfaces used as the prime mover to extract power. A theoretical exploration of array layouts is given in Draper et al. [19].

Tank testing of tidal arrays to date has been limited, due in part to the difficulty in finding appropriate testing facilities. Myers and Bahaj [20] investigated array layouts through porous disks in a shallow tank with the aim of maximising the flow acceleration through the array. Draper et al. [17] conducted a similar study with the focus on the evolution of the wakes. However, these studies investigated flow acceleration and not the extracted power. Cooke et al. [21] used the thrust on the disk along with a near wake velocity measurement to infer the power, finding power coefficients per disk of $\approx 0.1$ based on the global channel flow.

More complex flow effects caused by a dynamic turbine model (such as rotational effects) are not present in porous disk experiments. Stallard et al. [12] have investigated the layouts of up to ten three-bladed Horizontal Axis Turbines (HATs), with lateral spacings of $1.5 D$ and $2 D$ (where $D$ is a rotor diameter) over two rows. They showed velocity deficits of $80 \%$ across the turbines, $2 D$ downstream by which point the wakes had begun merging and were fully merged by $4 D$.The widths of the individual wakes were seen to expand to a maximum of $2 D$ by $10 D$ downstream. Power was measured via a dynamometer but the variation of power with array layout is not reported as the work focuses on the wake evolution.

Another HAT device study using two in-line three-bladed dynamic tur- 
bine models at the deeper (2m) IFREMER tank was presented by Mycek et al. [22]. They showed a drop in turbine performance in the downstream device compared with a single turbine for a series of downstream spacings. They also show that in high turbulence environments, increased downstream mixing leads to lower velocity deficits increasing downstream device performance suggesting that velocity magnitude is more important than turbulence.

\subsection{The Momentum Reversal Lift (MRL) turbine}

The momentum reversal lift turbine, shown in Figure 1, was conceived by the University of Exeter and Aqua Scientific Ltd. This cross flow horizontal axis turbine has three symmetrical blades, each of which rotate through $180^{\circ}$ for a full rotation of the shaft. The turbine is unique in that it utilises both lift and drag (momentum reversal) in order to generate rotational velocity in the prime mover. The turbine is designed primarily for shallow estuaries where the cross flow design will allow for high blockage ratios relative to a circular swept area device thus increasing power output. For a comprehensive overview of the turbine design see [4] and [7].

Initial experiments utilised both a balsa wood model in a wind tunnel and a metal turbine in a flume. These devices both showed promising maximum $c_{p}$ values of $\sim 0.5$, however these were in high blockage environments, 0.66 in the case of the flume [4]. The flume results were compared with a Immersed Body Force (IBF) CFD model utilising Large Eddy Simulation (LES), which showed good similarity with the experimental data, particularly for the lower torque range $[6,7]$.

The scale model turbines used here were previously tested in a wider flume at the IFREMER facility where some initial array configurations were 


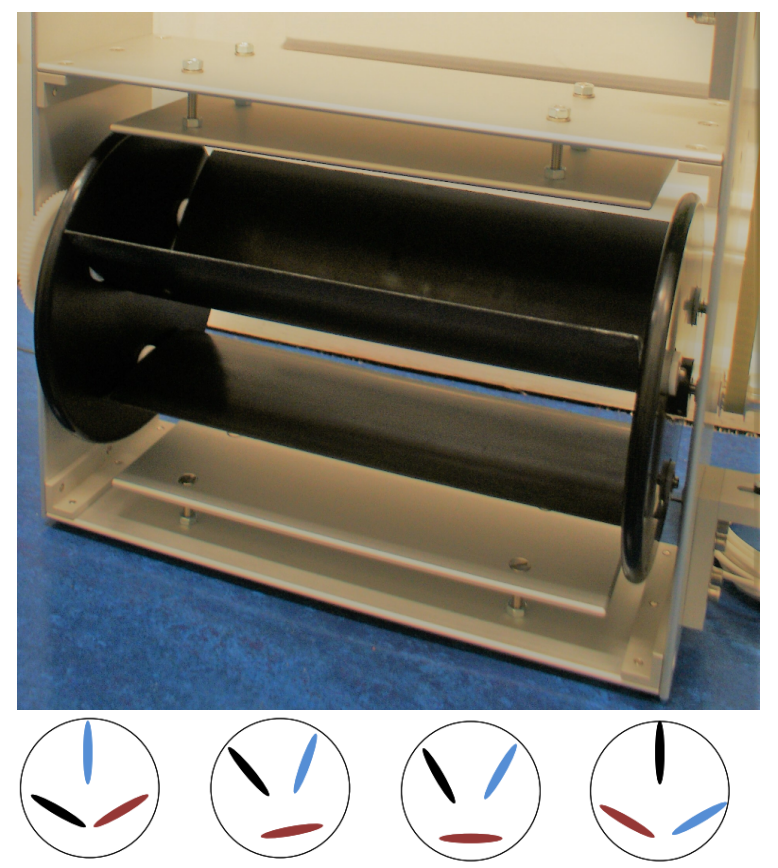

Figure 1: MRL turbine prototype used in the experiments described in this paper (top). The three bladed rotates around the shaft as it can be observed in the bottom figure. In the initial set up one blade is set completely flat while the others two start with a set angle. 
Table 1: Array spacings in multiples of turbine diameters (D) and $c_{p}$ of the centre turbine from CFD [8].

\begin{tabular}{|c|c|c|}
\hline $\begin{array}{c}\text { Downstream } \\
\text { spacing }\end{array}$ & $\begin{array}{c}\text { Lateral } \\
\text { spacing }\end{array}$ & $c_{p}$ \\
\hline $10 \mathrm{D}$ & $3 \mathrm{D}$ & 0.40 \\
$10 \mathrm{D}$ & $6 \mathrm{D}$ & 0.30 \\
$15 \mathrm{D}$ & $3 \mathrm{D}$ & 0.45 \\
$15 \mathrm{D}$ & $6 \mathrm{D}$ & 0.32 \\
\hline
\end{tabular}

trialled [5]. The $c_{p}$ values in a relatively open channel dropped to 0.14 . In these tests, the wake had not recovered to the upstream velocity magnitudes by $20 \mathrm{D}$ downstream. In addition there was evidence of asymmetry in the wake of a single turbine which is not evident in similar three bladed HAT testing [12].

There have also been studies using the IBF model to investigate trends in array layouts. These have investigated both changing spacings [8] and varying resistive body force [9]. The former looked at a test matrix of two lateral and two downstream turbine separations for three rows of turbines in a 2-3-2 formation, with a blockage ratio of 0.044 . Table 1 presents the spacings and $c_{p}$ values. It found the highest $c_{p}$ values were in the narrower (lateral) and longer (stream-wise) arrangement, with an increase in lateral spacing causing a decrease in $c_{p}$. In addition, while it was found that the blockage of the first row of turbines caused an acceleration into the middle turbine in the second row, this did not necessarily mean an increase in $c_{p}$. 


\subsection{Aims and objectives of current study}

A key goal in tidal energy research is to determine the extent to which array layouts affect the extractable power. Arrays can be staggered or in-line and the lateral and downstream spacings between devices can all be varied.

In this work five array layouts are trialled with the aim of maximising power output and exploring the extent of the influences of spacing and layout on both power and flow. The evolution of the wake is also investigated through two sets of streamwise lines of velocity measurements, to allow comparison with previous studies. Array layouts are chosen to highlight the effect of changing a single metric and to align with previous CFD modelling work.

\section{Test Set-up and Methodology}

\subsection{Overview of the FloWave test tank}

The array testing was conducted at the FloWave Test Tank facility. This $25 \mathrm{~m}$ diameter circular tank has the facility to provide combined wave and current, with wavemakers located around the entire circumference. The nominal test area has a diameter of $10 \mathrm{~m}$. The tank is capable of generating currents upwards of $1.6 \mathrm{~ms}^{-1}$, using 28 drive units mounted in a plenum chamber below the test floor. Turning vanes mounted below and in front of the wavemakers direct the current across the tank. These turning vanes incorporate porous screens to provide flow conditioning and prevent debris ingress to the plenum chamber [23]. This facility was selected due to the large test area required for array testing.

In order to create an approximately uniform current across the test area of the circular tank, the impeller units on either side of the required current 
direction (i.e. both the upstream and downstream) are utilised. These are driven at varying speeds to produce the required current corresponding to the desired test velocity. This results in an 'hour-glass' shaped flow profile in the $x y$ plane [24]. Previous measurements in the tank have shown the flow to be highly symmetrical about the stream-wise $(x)$ axis [25]. However, in the streamwise direction there is some variation in both the mean and turbulent flow parameters. The velocity varies approximately linearly with depth but has a very shallow gradient compared with measurement at full scale sites $[26]$.

\subsection{Turbine Models}

The small scale model utilised here is shown in Figures 1(bottom) and 2. The model has three $300 \mathrm{~mm}$ wide $(L)$ and $95 \mathrm{~mm}$ chord length blades mounted on a planetary gear system. The distance from the primary shaft to the centre of rotation of each blade is $164 \mathrm{~mm}$. The cross sectional height of the turbine $(D)$ is $200 \mathrm{~mm}$ giving a 'swept area' $(A)$ of $0.06 \mathrm{~m}^{2}$. Note that this cross sectional area is not entirely swept by the blades due to the change of angle through the rotation, but since the design prohibits mounting another device within this cross sectional area the adopted definition was deemed the most appropriate. Ground force and Pelton effect plates, which act to increase the flow rate through the swept area, were added during early testing in order to increase rotational velocity [4]. Power take off is provided by a $2.5 \mathrm{kSt}$ oil-filled dash-pot connected to the primary shaft by a $2: 1$ geared pulley and the angular velocity $(\omega)$ is measured via a 24 tabbed disk mounted on the primary shaft which passes through a Hall effect sensor. Part of the previous work focused on finding the gear ratio and damper which produced 


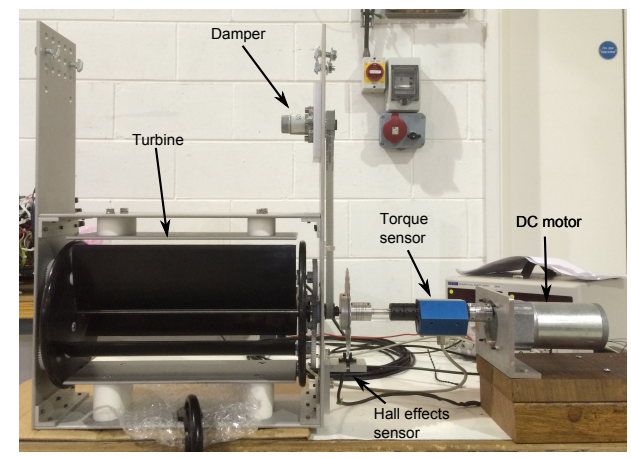

Figure 2: Model used in testing attached to torque calibration rig.

the highest power coefficient $\left(c_{p}\right)$ [5].

The scale models utilised in these tests were by necessity relatively inexpensive to allow a relatively large number to be constructed and as such, there was some variation in angular friction from one turbine to the next. This is detailed further in Section 4.1. Therefore, it was necessary to calibrate the torque for each individual turbine and its damper for a range of rotational speeds. The rig for calibrating this torque curve is shown in Figure 2. It features a $2 \mathrm{Nm}$ rotary torque transducer attached to the primary shaft and the system is driven by $27 \mathrm{~W}$ DC motor. For each turbine a measurement was taken every $2 \mathrm{~V}$ from $4 \mathrm{~V}$ to $24 \mathrm{~V}$ which provided a range or rotational speeds up to approximately $140 \mathrm{rpm}$. At each setting the rotational velocity $(\omega)$ and torque $(\tau)$ were collected via a National Instruments data acquisition system and recorded via Labview.

In order to mount the turbines in the tank, an adaptable frame design was developed that would allow for relatively quick changes between array configurations. Ideally the turbines would have occupied a greater percentage of the channel depth increasing the global blockage ratio. However, with the 

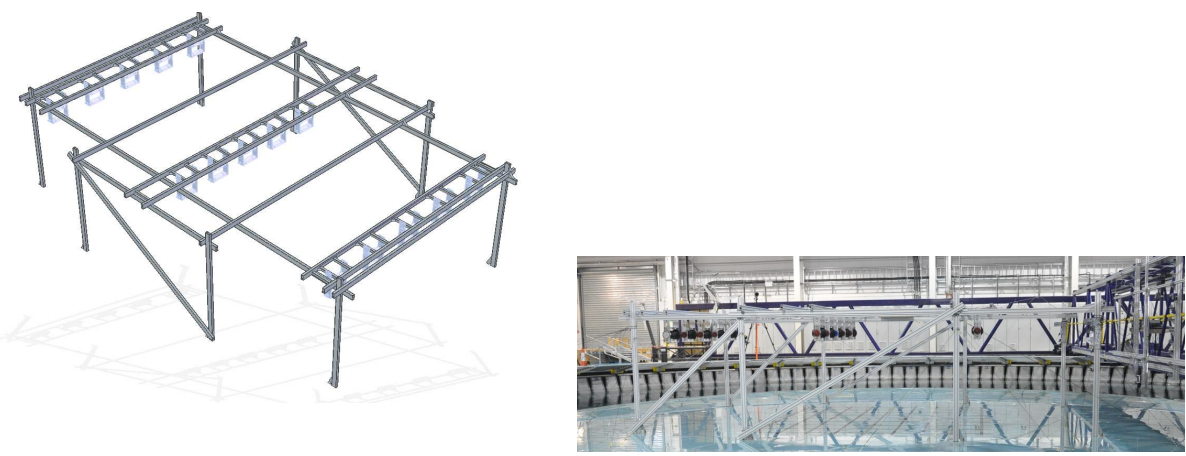

Figure 3: The frame for mounting the turbines, which was designed for high stiffness and for ease of reconfiguring turbine locations.

working depth of FloWave being $1.9 \mathrm{~m}$ this was not possible. The turbines were mounted close to the free surface as this would provide realistic blockage effects at one boundary as per the design specification [6]. Figure 3 shows the frame which was constructed from $45 \times 90 \mathrm{~mm}$ cross section aluminium extrusion and Figures 5 and 6 highlight its location in the tank. In order to increase the rigidity of the frame, guy lines were tied to the tank floor from each of the vertical poles. In order to track any vibration of the frame and also the position of the turbines, a Qualisys tracking system was employed. This showed that under load the maximum movement in the frame was $<4 \mathrm{~mm}$.

\subsection{Array Layouts}

Five different array layouts were tested in order to assess the effect of: lateral separation, stream-wise separation and in-line and staggered rows of turbines. To this end a base-case array layout was selected. Each of the other arrays vary one parameter from this base-case. The spacing are relative to the turbine dimensions where $D$ is the cross flow height (equal to 200mm) and $L$ is the cross-stream width (equal to $300 \mathrm{~mm}$ ). 
Table 2: Overview of the array layout edge to edge spacings used in the tests. Bracketed numbers indicate the number of devices in each row.

\begin{tabular}{llcc}
\hline $\begin{array}{l}\text { Layout } \\
\text { code }\end{array}$ & $\begin{array}{l}\text { Staggered } \\
\text { /In-line }\end{array}$ & $\begin{array}{c}x \\
\text { spacing }\end{array}$ & $\begin{array}{c}y \\
\text { spacing }\end{array}$ \\
\hline Baseline & Staggered (4-5-4) & $14 D$ & $2 L$ \\
A & Staggered (4-5-4) & $10 D$ & $2 L$ \\
B & Staggered (4-5-4) & $14 D$ & $1.5 L$ \\
\hline C & In-line (5-5-5) & $14 D$ & $2 L$ \\
D & In-line (5-5-5) & $14 D$ & $1.5 L$ \\
\hline
\end{tabular}

Each of the arrays featured three rows of turbines. All of these configurations included five turbines in the middle row. The staggered layouts had four turbines in the front and back with these spaced at the mid points in the transverse $(y)$ between the middle row turbine locations. For the in-line arrays the turbines were mounted in the same $y$ locations in each of the three rows, each containing five devices. The staggered arrays featured 13 turbines and the in-line 15 devices to accommodate this. Table 2 provides an overview of the array configurations which were investigated.

\subsection{Measurement strategy}

In addition to the rotational velocity of each turbine, the flow velocity within each array was investigated. In order to do this a Nortek Vectrino was utilised to measure the flow velocities. This instrument is capable of measuring at $100 \mathrm{~Hz}$ resolving the velocity into three Cartesian components. The Vectrino was mounted to the tank's instrumentation gantry on an adjustable frame, allowing it to translate in the $x, y$ and $z$ directions. As with any 
acoustic sensor, measurements are subject to uncertainty due to noise. This Doppler noise is generally agreed to be zero mean, thus mean velocities are computed over a large number of samples (6000) to reduce the uncertainty. This noise will bias the turbulence intensity value high but this is corrected for by measuring the variance due to noise using the method described by Richard et al. [27].

To maximise the extent of velocity information from throughout the array, symmetry about the $x$ axis was assumed, based on research that showed the undisturbed tank flow to be symmetrical [25] and that the frame and turbine array layouts were also symmetrical. All measurements were taken at a fixed depth at the midpoint of the turbine swept area. The co-ordinates for measurements are given in terms of their $(x, y)$ position in $\mathrm{mm}$ from the centre point of the tank, where $x$ is positive upstream of the centre.

For each array, one measurement $\left(u_{0}\right)$ was taken significantly far upstream as to not be affected by the array as a reference. From there, one measurement was taken $2 D$ upstream of each turbine on the $y \leq 0$ side of the array, this is referred to as the $u_{i n}$ measurement. In addition the development of the flow along the $x$ axis is measured at $y=0$ and at $y_{\text {spacing }} / 2$. These where taken every $2 D$ downstream (or as close as possible where the horizontal frame beams were obstructing access) from the first row to the last row of turbines. The positions of the array relative to the stream-wise direction of the tank were varied to minimise effects of the support struts on the flow.

Tests were carried out at the nominal, scaled current speed of $1.2 \mathrm{~ms}^{-1}$, although higher speeds were used on occasion to ensure all turbines cut-in, before being reduced to $1.2 \mathrm{~ms}^{-1}$ again for testing. 
Each velocity vector is the result of one minutes measurement. This magnitude has previously been shown to be a statistically stationary period [26] and a mean value for each vector (e.g. $\bar{u}$ ) is reported. In addition to this, other metrics are used to give more information about the behaviour of the flow: the mean stream-wise velocity deficit $(\Delta u)$, the turbulence intensity for each vector (i.e., $I_{u}$ ), the heading $(\theta)$, and the pitch $(\psi)$ which are defined in the following equations:

$$
\begin{gathered}
\Delta u=100 \cdot\left(1-\frac{\bar{u}}{u_{0}}\right) \\
I_{u}=\frac{\sqrt{\sigma_{u}}}{\bar{u}} \\
\theta=\tan ^{-1}\left(\frac{\bar{v}}{\bar{u}}\right) \\
\psi=\tan ^{-1}\left(\frac{\bar{w}}{\bar{u}}\right)
\end{gathered}
$$

\subsubsection{Power}

In order to compare the different arrays an appropriate metric must be defined. For the individual turbines the power extracted is given in Equation 5 , where $\omega$ is the angular speed (in $\mathrm{rad} / \mathrm{s}$ ) and $\tau$ the torque of the turbine at that angular velocity calculated via the calibration curve detailed in section 4.1. The local power available in the flow (i.e. availably to a specific device) is defined via Equation 6, where $\rho$ is the fluid density and $A$ the swept area of the turbine. 


$$
\begin{aligned}
P_{\text {turbine }} & =\omega \tau \\
P_{\text {available }} & =0.5 \rho A u_{i n}^{3}
\end{aligned}
$$

This allows the calculation of the power coefficient of the turbines, defined via Equation 7:

$$
c_{p}=\frac{P_{\text {turbine }}}{P_{\text {available }}}
$$

Note that the central goal of this study is to maximise power output. Thus, in order to compare the power captured by each of the array layouts, three power metrics are used: $P_{\text {total }}$ i.e. the sum of the mean power from each turbine, $P_{\text {mean }}$ the mean output from each individual turbine and finally $\frac{P_{t o t a l}}{m^{2}}$ the power per square meter of the array based on the total $x y$ 'footprint' area of the array configuration.

For tidal and wind turbines the power is often expressed as a function of the Tip Speed Ratio which is the ratio of the velocity at the blade tip to the velocity of the inflow fluid. As the MRL turbine tip speed is difficult to define, here the Blade Speed Ratio $(B S R)$ is used as an equivalent. $B S R$ as defined in [5] as:

$$
B S R=\frac{\omega R}{u_{i n}}
$$

were $\omega$ is the angular velocity in $\mathrm{rad} / \mathrm{s}$ and $R$ is the radius to the axis of blade rotation.

\subsection{Scaling and Blockage}

As there is no prototype scale device against which to scale the MRL turbine, the depth and flow speeds of a typical tidal site of $50 \mathrm{~m}$ and $3 \mathrm{~ms}^{-1}$ 
are considered respectively. These values do not represent estuary conditions, but given the depth of the test tank relative to the model turbine, this was deemed appropriate for this test, where the tank depth is significantly greater than the turbine diameter.

The two main scaling factors in tidal arrays are the Reynolds number, a ratio of the momentum to viscous forces, and the Froude number, a ratio of the inertia to the gravitational effects on the flow. These ratios are defined in equations 9 and 10 where $\rho$ is the density, $l$ is a characteristic length and $g$ is the gravitational field strength [17]. For the same fluid and gravitational forces these two dimensionless quantities can not be equally scaled. However, flow conditions are required to be within the same regimes, i.e., fully developed turbulence and sub-critical [28].

$$
\begin{aligned}
& R e=\frac{\rho u l}{\mu} \\
& F r=\frac{u}{\sqrt{g l}}
\end{aligned}
$$

If $l$ is taken to be the channel depth and $g$ is taken to be constant at $9.81 \mathrm{~ms}^{-1}$, the $R e$ and $\mathrm{Fr}$ numbers for this test and for the nominal site are given in Table 3 . These numbers are in the range of those in similar work $[17]$.

Whilst some authors have envisaged that Froude number has minor influence in power and thrust (both increase about 3\% according to [29]), the discrepancy between Reynolds numbers between prototypes and full scale devices has large effects in the performance of a tidal turbine. In Mycek et al. [30], Reynolds numbers from $1.4 \times 10^{5}$ to $4.2 \times 10^{5}$ were used in the 
Table 3: Comparison of scaling parameters

\begin{tabular}{|c|cc|}
\hline & $R e$ & $F r$ \\
\hline FloWave & $2.4 \times 10^{6}$ & 0.32 \\
Full Scale & $1.3 \times 10^{8}$ & 0.09 \\
\hline
\end{tabular}

experimental campaign. It was demonstrated that the $c_{p}$ of a turbine can increase by about $10 \%$ when working at larger flow velocities and hence larger Reynolds numbers. This increase is somehow to be expected, Mason-Jones et al. [31] suggested that in order to reach Reynolds independence, Reynolds numbers higher than $3 \times 10^{5}$ should be contemplated in small scale test campaigns. However, this insensitivity of Reynolds number could be dependent on the aerofoil shape but according to the authors knowledge there is no evidence to prove it. As it has been envisaged by Selig et al. [32], wind tunnel tests have demonstrated that the magnitude of lift on thick aerofoils can be increased slightly when increasing Reynolds numbers from $1 \times 10^{5}$ to $5 \times 10^{5}$. However, the effects on drag will be severely, in some cases an increase of $50-80 \%$ was observed at angles of attack between 0 to 10 degrees. This proportion depends on the type of aerofoil shape, in this case the S822 was taken as an example. Wind tunnel tests studies will need to be considered in the next development stages of the MRL turbine due to the constant and different changes in angle of attack related to each of the blades.

As previously stated the blockage for this test is relatively low compared with early tests of this device and other arrays. The swept area of the staggered arrays are $9 \times A$ which is: $0.2 \times 0.3 \times 9=0.54 \mathrm{~m}^{2}$. The tank area at the mid point as $25 \times 1.93=48.25 \mathrm{~m}^{2}$. This leads to a global blockage 
ratio of 0.010 for the staggered arrays and of 0.005 for the in-line arrays with a swept area of $5 A$.

\section{Results}

\subsection{Turbine Calibration}

The calibration results from each of the turbines with dampers installed are presented in Figure 4. The dashpot's resistance changes with temperature which is related to $\omega$ hysteretically. Thus, the calibration was repeated in ascending and descending $\omega$ to capture this effect. As can be seen, there is a spread of values with a maximum difference at the highest voltage used of $0.26 \mathrm{Nm}$ indicating a significant degree of variation in damping between devices. It can be noticed that the turbine 3A, which used an older damper of the same specification, showed the lowest resistance, indicating that the performance of these devices in this installation were decreasing over time or with use.

Multiple types were trialled to this data (using the downward calibration curve as all turbine measurements were taken at established speeds). The power law gave the highest goodness of fit values thus this fit type was adopted. The form of this curve is given in Equation 11, where $a$ and $b$ are constants defined individually for each turbine.

$$
\tau=a \cdot \omega^{b}
$$

\subsection{Base-Case}

As a large quantity of data was collected for each array, greater detail is provided for the base-case layout, which will provide values which can be 


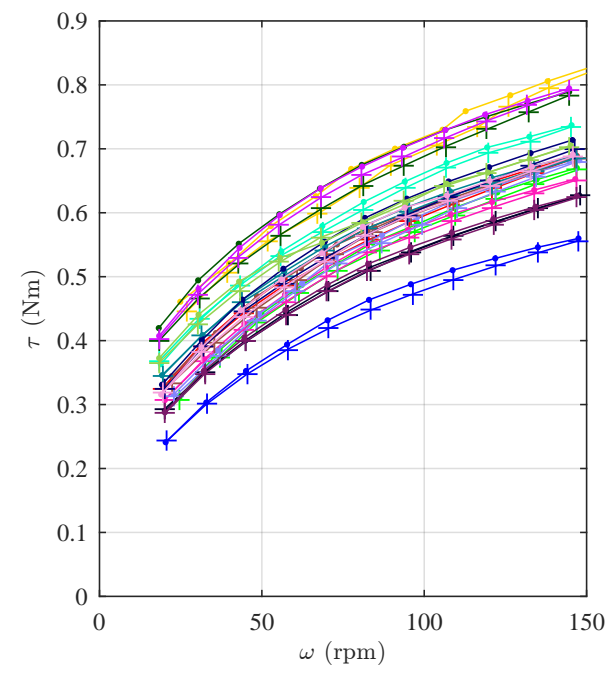

- 3A - N02• N04• N06• N08• N10 - N12• N14

Figure 4: Results of individual turbine torque calibrations. Dots mark the curve for increasing $\omega$ and crosses for decreasing.

contrasted with other layouts.

Figure 6 shows the positions of the turbines, the positions of the vertical pillars, the velocity measurements taken during the test, as well as the velocity measurements in the tank with no devices installed taken from Noble et al. [25]. This gives an illustration of where measurements were taken and the effect of the turbines on the flow velocity. These measurement locations do not capture the effects of the vertical support poles of the frame on the flow during the testing, which were observed, visually, to be significant.

\subsubsection{Velocity deficit and turbulence intensity}

Focusing on the two sets of stream-wise velocity measurements, Figure 7 shows the evolution of the flow through the turbines. As can be seen there is 


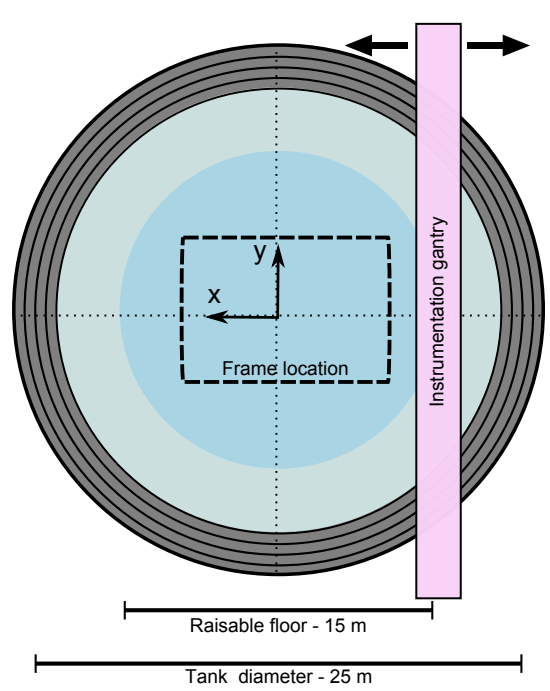

Figure 5: Illustration of test area, detailed in Figure 6, in dotted line and its position within the FloWave tank. Co-ordinate system is given from the tank centre.

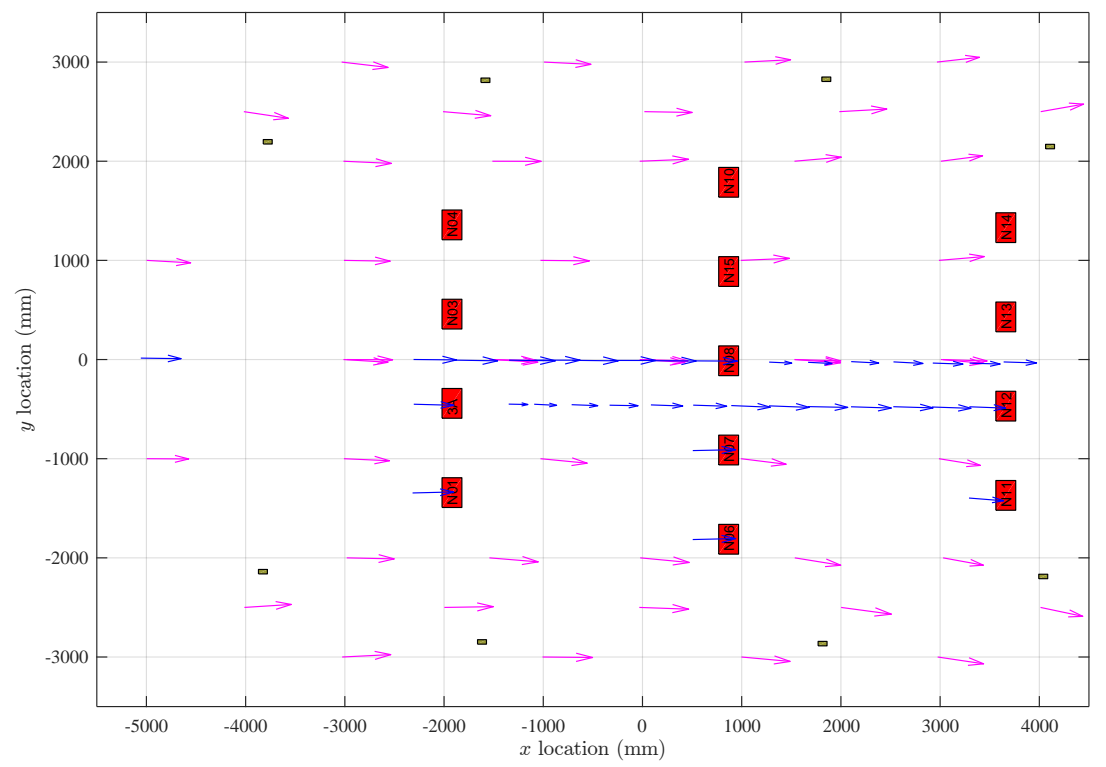

Figure 6: Quiver plot for the base-case array. Blue arrows represent in-situ velocity measurements, the pink arrows represent the 'natural' flow in the tank were there no obstructions. The large red rectangles represent the turbines and the small grey ones the vertical frame poles. 

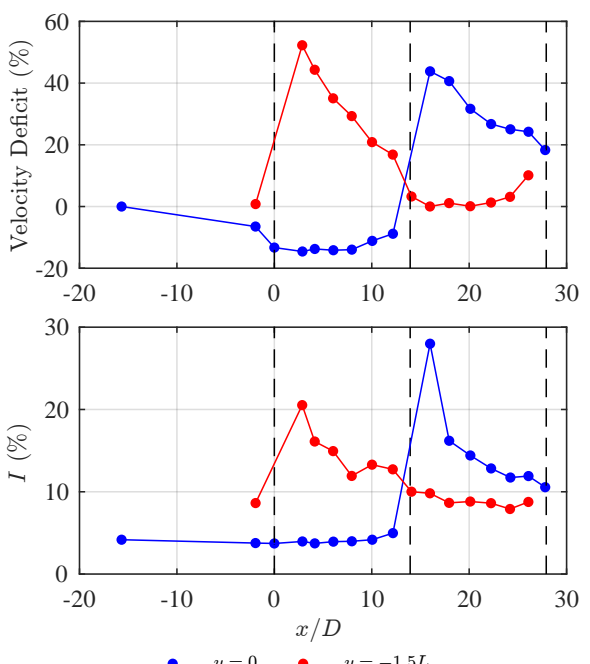

Figure 7: Velocity deficit and turbulence intensity of flow as it propagates through the base-case array. The dashed lines indicate the location of the turbine rows. Flow moving from left to right.

an acceleration from the upstream measurement point due to the tank flow geometry then an acceleration through at the centreline through the first row of turbines. There is a large decrease in velocity of $52 \%$ as the flow passes through a turbine with a similar decrease at $y=-1.5 \mathrm{~L}$ over the turbine in the middle row of $54 \%$. There is a corresponding jump in turbulence intensity at each row of $12 \%$ and then $26 \%$. It is interesting to note that, although the velocity deficits are approximately equal, in each case the downstream jump in $I$ is significantly greater. Downstream of the turbines, the velocity and $I$ start to recover towards their upstream values.

\subsubsection{Velocity direction}

A phenomenon which became apparent during testing was a standing surface wave downstream of the first row (Figure 8). This effect had been 


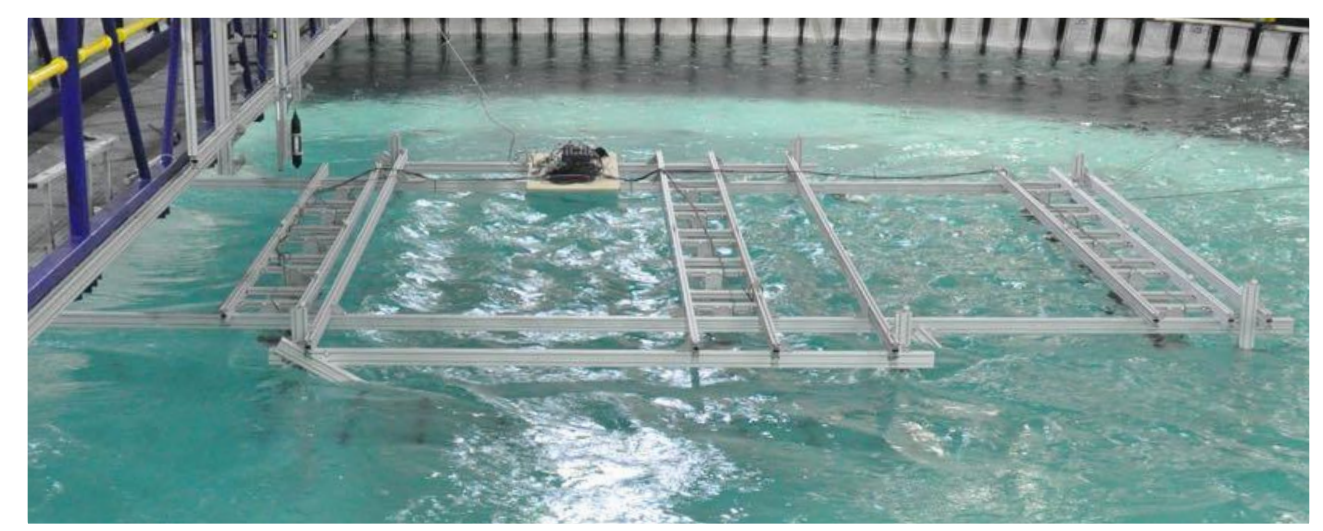

Figure 8: Evidence of 'standing wave' effects in wake. Flow moving from left to right.

previously noted in other experiments and predicted by CFD [17, 4].

The pitch and heading angles as the flow propagates through the rows of turbines are presented in Figure 9. The heading angles are relatively small $\left(<5^{\circ}\right)$ throughout. In the majority of cases, there is a $\sim 4^{\circ}$ shift of the flow to the left each time the flow passes through a row of turbine. This was only observed for the centre-line measurements in the base-case array. The measurements of the pitch angle of the flow are an order of magnitude greater than those of the heading. The measurement resolution is not high enough to capture properly the sinusoidal pattern visually observed. However, there is evidence of this effect between the first and second turbine rows. Each time the flow passes the turbine there is an upward shift in the pitch angle, which corresponds to the rotational direction of the turbine. The flow in the main has a small negative pitch angle, i.e. a downward trajectory.

\subsection{Turbine Performance}

The mean and standard deviation of individual turbine $\omega$ values for the base-case array are given in Figure 10. A large variation in values which 

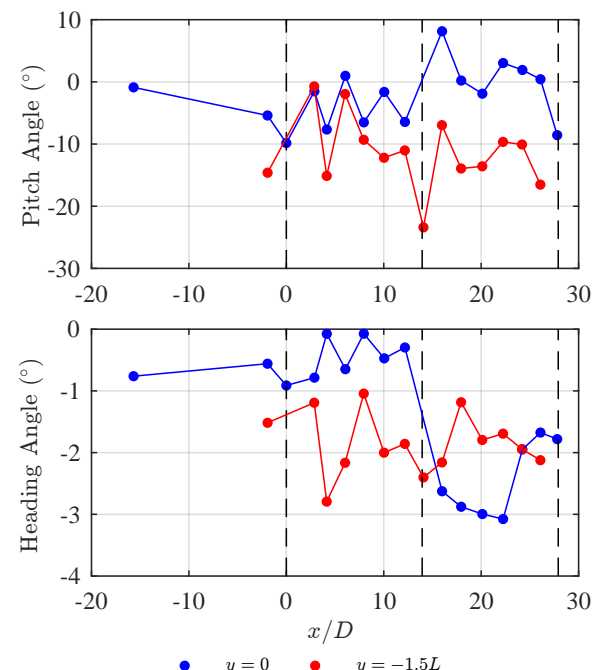

Figure 9: Results of velocity direction through the base-case array. Flow moving from left to right.

is due to the variability of both inflow velocity through the array and the damping of the individual turbines can be seen.

In order examine the variation of damping between devices, the angular velocity is converted to a $c_{p}$ value through equations $5,6,7$ and 11 . Limiting the results to those for which the inflow is directly measured (rather than inferred through the assumption of a symmetrical array) the $c_{p}$ for the Blade Speed Ratio $(B S R)$ is shown in Figure 11. This analysis uses Array A as there was an additional inflow measurement available.

It can be seen that the $c_{p}$ values for the turbines in the front and middle rows are approximately linear on a positive gradient, suggesting power capture increases as turbine damping decreases. However, the two back row values do not conform to this trend. These results suggest that the downstream turbines with inflows from the wakes of the upstream turbines are 


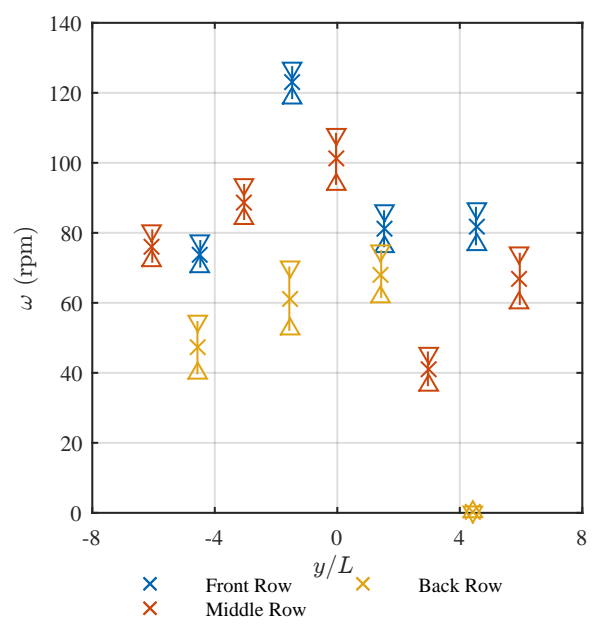

Figure 10: Base-case array mean and standard deviations of $\omega$.

less predictable than those upstream.

The $\overline{u_{i n}}, \bar{\omega}$ and $\bar{P}$ for each turbine in the base-case array are presented in Figure 12. It is reiterated that the $\overline{u_{i n}}$ measurements are only measured for half of each array layout and the $y>0$ values are inferred by assuming symmetry about the $x$ axis. One aspect that is evident is the lack of symmetry in the measured $\omega$ and $P$ values. In the top plot it can be seen that the middle row has the highest inflow velocities due to the blockage of the flow through the upstream turbine row. However, this higher $u_{i n}$ does not correspond to higher rotational velocities in the middle plot. As the turbulence intensity is also constant into both rows it is inferred that it is the vertical component of the velocity (i.e. the pitch of the flow as per Figure 9) that is affecting the reduction in $\omega$ in the middle row devices. The power is highly variable between devices in the two front rows, with the back row presenting the lowest variation and generally lowest response. 


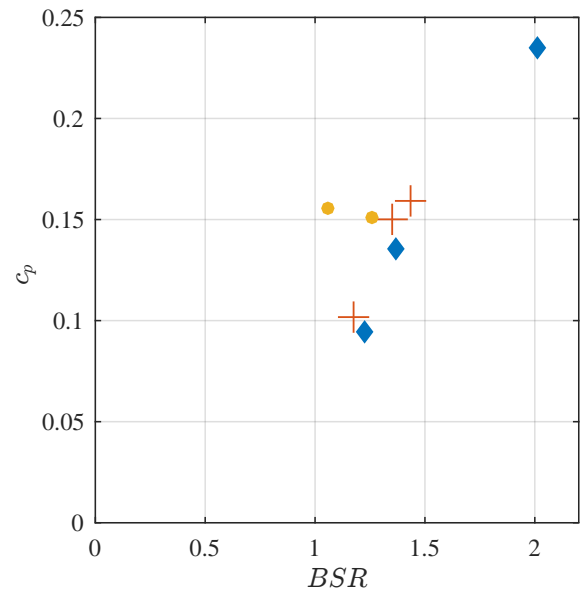

$\downarrow$ Front Row + Middle Row - Back Row

Figure 11: $c_{p}$ variation between turbines for array A.
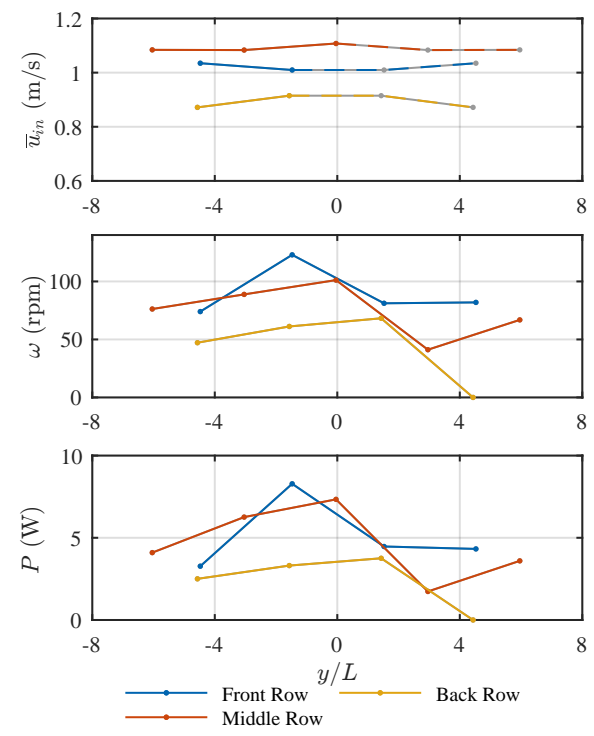

Figure 12: $\overline{u_{i n}}, \bar{\omega}$ and $\bar{P}$ for each turbine in the base-case array. 


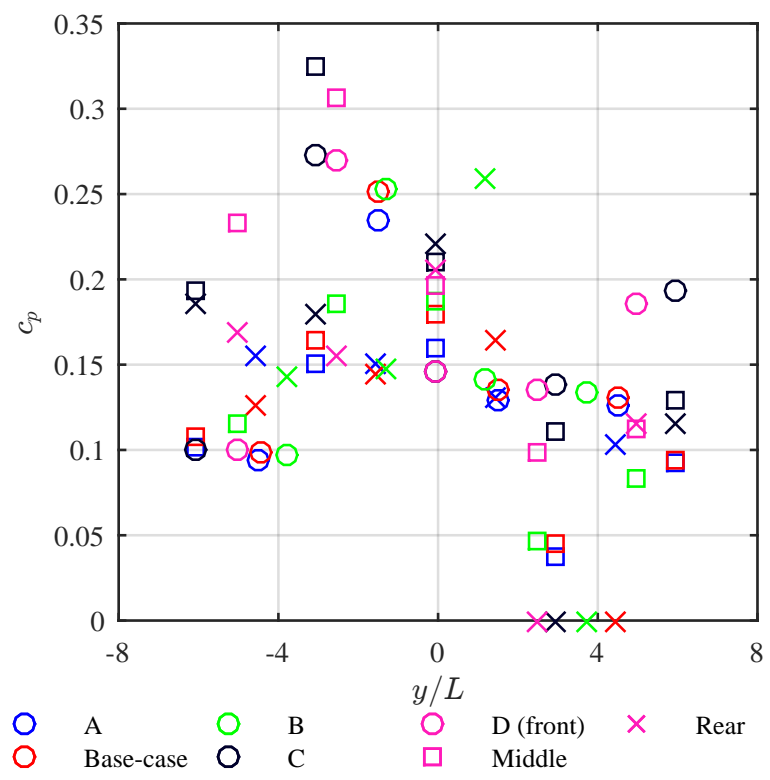

Figure 13: All $c_{p}$ values across the 5 arrays. Circles mark the front row, squares the middle and crosses the rear row, with colours indicating the array layout.

\subsection{Inter Array Comparisons}

The $\overline{u_{i n}}, \bar{\omega}$ and $\bar{P}$ values for each turbine for the four additional array layouts are given in Figures 14, 15, 16 and 17, with the base-case included in grey for comparison.

For the decreased stream-wise separation (Figure 14) the trends are very similar to those in the base-case array, with highest flow velocities being into the middle row of turbines due to acceleration between the first row turbines and similar $\omega$ values. In the decreased lateral spacing case (Figure 15) the velocity into the second row is very similar to the front row, suggesting that at this spacing the first turbine row as a whole has a blockage ratio that is causing more flow to divert round the sides of the array. The reason of this diversion is because the flow has reached its maximum choking capacity 

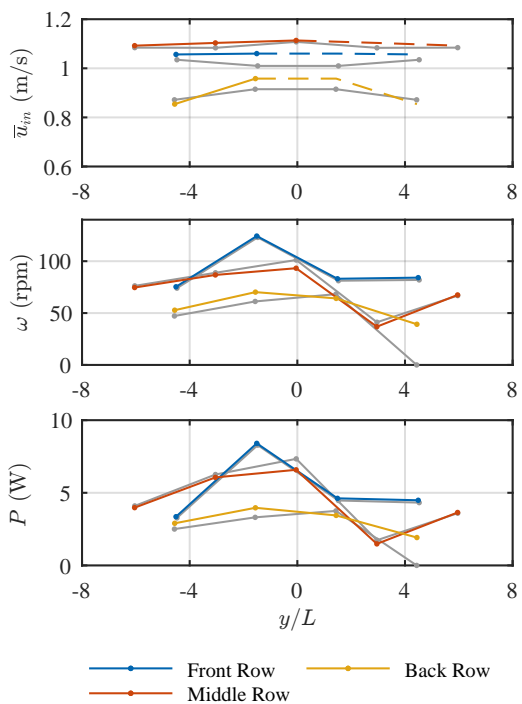

Figure 14: $\overline{u_{i n}}, \bar{\omega}$ and $\bar{P}$ for array layout $\mathrm{A}$, with base-case results in grey.
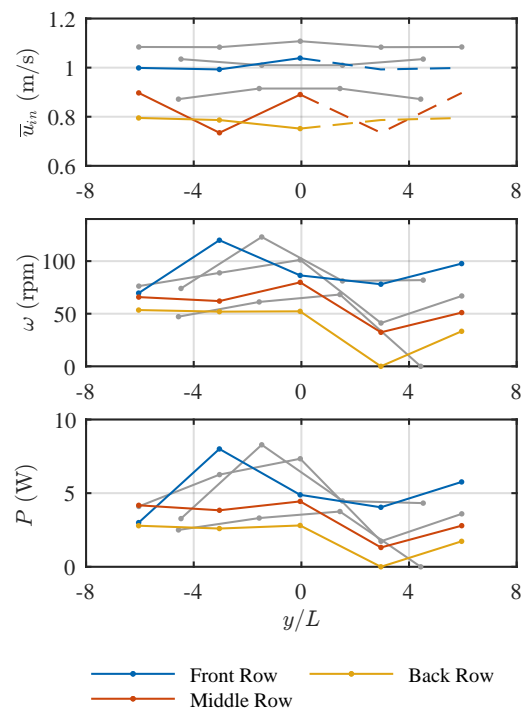

Figure 16: $\overline{u_{i n}}, \bar{\omega}$ and $\bar{P}$ for array layout $\mathrm{C}$, with base-case results in grey.
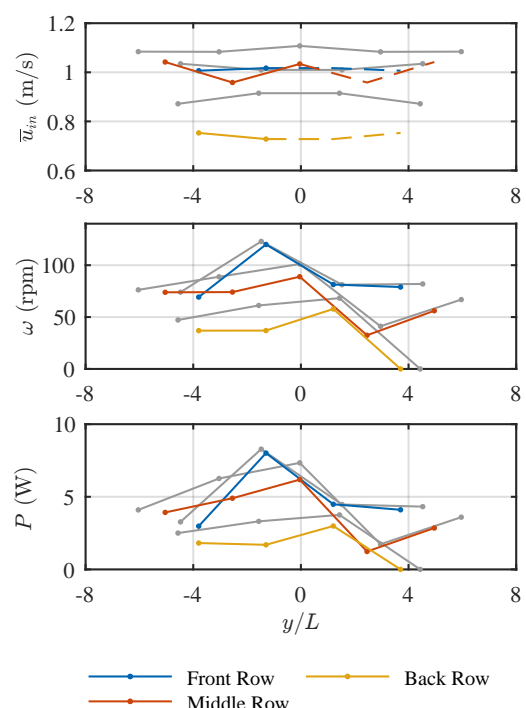

Figure 15: $\overline{u_{i n}}, \bar{\omega}$ and $\bar{P}$ for array layout $\mathrm{B}$, with base-case results in grey.
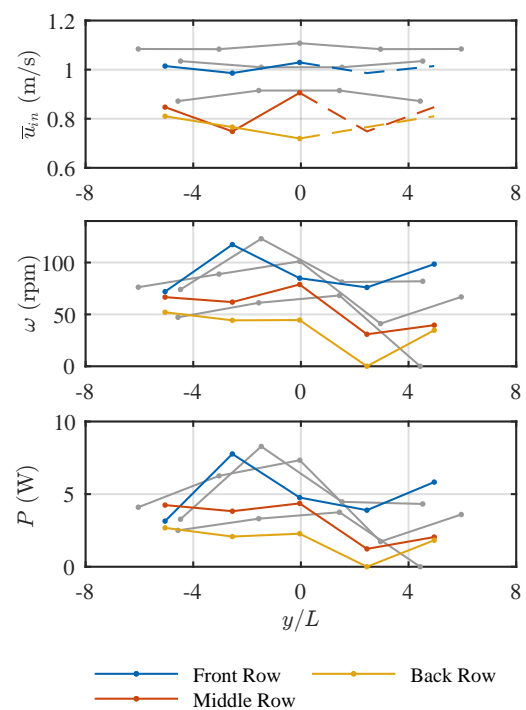

Figure 17: $\overline{u_{i n}}, \bar{\omega}$ and $\bar{P}$ for array layout $\mathrm{D}$, with base-case results in grey. 
which as defined by Nishino and Willden [15], is the reduced ow through an entire array. For all three of the staggered arrays there is little asymmetry of $P$ and $\omega$ results around the stream-wise axis in any of the rows. The trends in these metrics are, however, consistent between the three staggered array layouts.

In the two in-line arrays (Figures 16 and 17), $u_{i n}$ is highest in the front row as would be expected. However, there is evidence of the wake from the upstream posts affecting the inflow to the middle row at the second turbine from each edge in both array spacings, leading to those turbines having lower $u_{\text {in }}$ than the back row. This is not directly reflected in the $\omega$ and $P$ results which decrease row by row.

This asymmetry of power and angular velocity across the 5 arrays (seen in Figures 14 to 17), may in part be due to the direction of the flow in the tank which forms an hour glass shape [26]. Although through the majority of the test area the flow is uniform, at the edges a combination of this inward flow and the wake of the vertical poles may cause flow velocities from different directions to affect the turbines. However, the effect is relatively constant across the arrays suggesting that power comparisons are valid.

It has been observed in the literature that there are some effects on the power captured depending on the direction of the flow. However, Figure 9 shows that the heading angles in these tests are less than 4 degrees and therefore it was envisaged that such small angles will not have a significant impact in the power calculations. This supposition was due to the research presented by Galloway et al. [33] who showed that power reductions only become apparent with heading angles above 7.5 degrees. Also, the turbulence 
intensities at the outer turbines are greater than that at the $u_{i n}$ locations for the inner turbines, with range of values increasing from $4-14 \%$ to $14-25 \%$. Figure 18 shows how the velocity deficit and $I$ values vary in the in-line Array C. For the first and second turbine row there is a $\Delta u$ of $51 \%$ and $45 \%$ respectively, with a recovery to within $13 \%$ of the upstream value by $12 D$. The sharp peak in $I$ midway between the front and centre row exists at both spacing but it is not clear what the driving factor is. It may be a mixing point for the turbine wakes, but the spatial resolution of velocity measurements is insufficient to analyse.

In order to assess which array is the best, three power related metrics are employed. The results of power for each configuration are given in Table 4. In terms of total power, four of the five arrays show similar values with the narrow staggered Array B being the lowest and the base-case the highest. The total power per device is dominated by the two $0.6 \mathrm{~m}$ spaced staggered arrays, which both have thirteen devices, two less that the in-line arrays. In terms of power for a given footprint-area, it is Array A (decreased streamwise spacing) that gives the best results.

Figure 13 shows all the $c_{p}$ values across the five array configurations. The highest value is 0.32 which is for the middle row of Array C. The mean value, discounting any non-rotating devices, is 0.15 . There is evidence of the asymmetry in the arrays with the values on the left $(y / L<0)$ being significantly higher than those on the right, which is relatively consistent across the array layouts. 

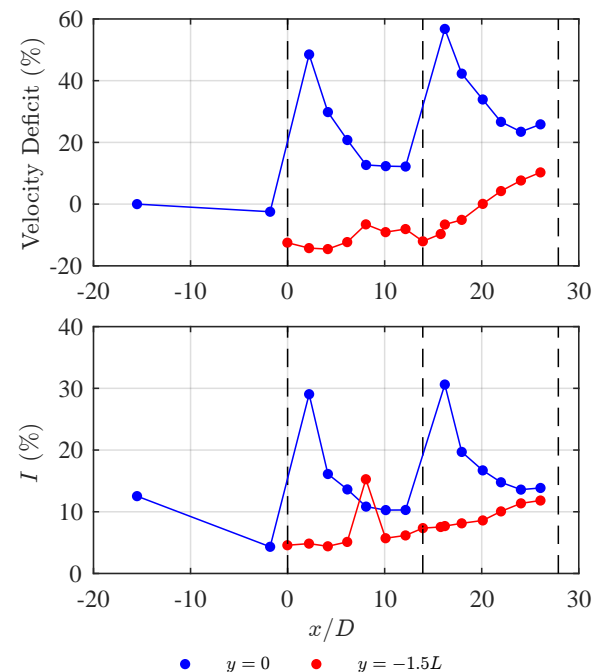

Figure 18: In-line array ' $\mathrm{C}$ ' showing the Velocity deficit and $I$ across two sets of turbines.

Table 4: Power parameters from each array layout.

\begin{tabular}{l|ccccc}
\hline & \multicolumn{5}{|c}{ Array } \\
& Base-case & A & B & C & D \\
\hline Total $P(\mathrm{~W})$ & 55.4 & 54.8 & 47.0 & 54.8 & 52.0 \\
$P$ per Device $(\mathrm{W})$ & 4.3 & 4.2 & 3.6 & 3.7 & 3.5 \\
$P$ per $x y$ & 2.8 & 3.8 & 2.8 & 2.7 & 3.1 \\
area $\left(\mathrm{W} / \mathrm{m}^{2}\right)$ & & & & & \\
\hline
\end{tabular}




\section{Discussion}

The three power metrics show that Array A has the highest power per footprint area and the base-case the highest total power. Comparing the trends from the base-case with similar trends in the CFD work [7, 8]: the decreasing lateral separation had the opposite effect on power in the CFD study compared to the current experiments. It should be noted that in the experimental work these separations are $2 L$ and $1.5 L$ (due to practical limits of the tank) whereas in the CFD these are $2 L$ and $4 L$. Malki et al. [34] suggests that a lateral spacing of $1 D$ represents a critical ratio after which decreased lateral separation decreased the bypass flow. This reverse of trend between experimental and CFD results is likely due to the experiments operating in the region of this the critical array 'choking' capacity. Only two lateral separations were tested in this work and thus a useful follow up study would focus on finding the exact point of lateral separation for maximum downstream turbine power. Likewise the downstream separation will allow mixing to occur (decreasing $I$ and increasing $u$ at the inflow to downstream devices) but also requires a greater $x y$ area and there will likely be a maximum value of $P / A$ in this trade off.

The $c_{p}$ values recorded are similar to those in Ordonez et al. [5] but lower than earlier studies [4] and lower than for other small-scale turbine experiments [35] which both produced maximum values of $c_{p}>0.45$. It should be noted that the MRL turbines were designed for high blockage ratio flows and thus the $c_{p}$ results presented were expected to be lower than these high blockage tests. If a suitable facility could be found, a repeat test in shallow water, where the turbines could be mounted to the floor to avoid the 
effects of structural struts on the flow would be of benefit. In addition, work by Salter and Taylor [36] and Nishino et al. [15] has suggested that very high global blockage ratios would increase $c_{p}$ and allow the Lanchester-Betz limit to be exceeded. This is due to the tidal channel being more analogous to a duct than unbounded flow for which the limit was defined. This would require larger turbine models or a shallower tank to effectively test this hypothesis, which remains a key query for the industry.

An interesting wake effect was the standing wave at the free surface behind the first row of turbines. This effect was also noted in the results of [17], who speculated it could be due to critical bypass flow. However, with the Froude numbers in this experiment of 0.32 being significantly below the critical value of 1 , it is far more likely the effect is due to vertical mixing and head loss across as the flow travels through the turbine. This varying flow direction will change the effective angle of attack of the blades in downstream devices. There is likely to be a 'sweet-spot' within the wavelength of the standing wave that will improve efficiency of downstream devices. This will be at the angle relative to the blade acting primarily in lift (rather than drag) where the flow direction has the effective angle of attack of highest lift coefficient. It was also noted that the pitch angle had a small negative (downward) velocity throughout the measurements. This may in part be due to a small misalignment of the ADV in this plane.

A key source of uncertainty in this experimental work was the asymmetry in the turbines' in-flow velocity. A previous study validated the symmetry of the flow in facility and the turbines and the frame were mounted symmetrically about the centre line of the tank. Due to this the inflow to individual 
turbines was only conducted for half the devices, in order to maximise the number of arrays that could be trialled in the available test time. The results presented show that there are changes in flow heading as it passes through the arrays. While Ordonez et al. [5] observed evidence of asymmetry in the wake, no measurements were made of the transverse velocity components. Thus, no direct comparison can be made with the present tests and the measured transverse flow velocities. It is recommended for future tests that inflow measurements are taken for all devices. In addition advancing the models to a higher degree of sophistication including active torque or speed control could reduce variation of results between devices.

In addition to this, the wake from the support poles played a large role in the results. Different turbine array layouts were affected differently due to different turbine positions relative to these poles. While this increases the uncertainty of these results, it is also a reminder of the sensitivity of machine performance to likely complex local flows in a real field setting.

In summary there are three proposed optimising spacings for this type of turbine in arrays:

1. A stream-wise spacing to maximise $P_{\text {total }} / A$ of the order of multiple rotor diameters;

2. A lateral spacing to maximise bypass flow, i.e., the array 'chocking' capacity;

3. A refinement of the stream-wise spacing of the order of a single rotor diameter, to find the optimum performance within the standing wave wavelength.

It is still a point of debate as to which metric is best for comparing arrays. 
Power per $x y$ area is one logical choice but this is only worthwhile if the area constraint is likely to be the dominant parameter in the array design. This subject would require a site optimisation tool and is likely project specific.

As this work represents one of the largest array testing projects to use rotating models at this scale, there is significant scope for future work, beyond what has already been discussed in this section. An expansion to compare different turbine types such as conventional three-bladed HATs would inform differences in array spacings for different designs. It is obvious from the results presented that flows through arrays are complex and it is best to measure at as high a spatial resolution as resources allow. Finally, for many tidal sites the direction of flow varies significantly [10]. Hence a test of array sensitivity to off angle flow is a key metric to predict total power over a full tidal cycle.

As the first commercial arrays of devices are shortly to become a reality, the increased knowledge of flow interaction and array layout optimisation represent essential knowledge. However, with many key question still associated with a high level of uncertainty further work is needed to ensure the successful progression of the industry.

\section{Conclusions}

Comparing the power extracted for each trend in the arrays the following conclusions can be drawn:

- Power extraction changes the flow through the arrays, with the power per turbine varying by up to $19 \%$ in the arrays presented. Thus positioning turbines is important to maximise power output as predicted. 
- Increasing streamwise spacing increases the total power captured in the staggered arrays (base-case and A). This is as predicted by [16] who suggest that downstream rows will be less effected by the performance of upstream rows.

- Staggering rows generally improves the power per device as predicted by $[16]$.

- Decreasing lateral spacing can increase or decrease power output as there is an optimal local blockage to maximise power output [15]. The results here show a decrease in power output for both staggered and in-line arrays suggesting that the spacing in the narrow arrangements (arrays B and D) are at a spacing less than this critical array 'choking' capacity.

\section{Acknowledgements}

The authors would like to thank Nortek UK for the loan of the Vectrino Profiler for use in these experiments. We would also like to thank the following staff at The University of Exeter who worked so hard to support the model construction: Siobhan Kelley, Jordana Broom \& Steve Redshaw. The authors would also like to acknowledge the EPSRC for funding this work under grant number: EP/J010138/1.

\section{References}

[1] C. Legrand, Assessment of Tidal Energy Resource, Tech. rep., Black and Veatch, EMEC (2009). 
[2] Carbon Trust, UK Tidal Current Resource and Economics Study, Tech. Rep. July (2011).

[3] DECC, Digest of UK Energy Statistics: Chapter 5 Electricity (2015) 113-155doi:10.1227/01.NEU.0000028161.91504.4F.

[4] A. P. Janssen, M. R. Belmont, Initial research phase of MRL turbine. Technical report, Technical Report No: MO 562L.

[5] S. O.-S. D. S. G. S. P. T. B. M. G. M. R. B. I. Moon, Experimental Evaluation of the Wake Charecteristics of Cross Flow Turbine Arrays, Ocean Engineering.

[6] M. G. Gebreslassie, G. R. Tabor, M. R. Belmont, CFD simulations for investigating the wake states of a new class of tidal turbine, in: International Conference on Renewable Energies and Power, 2012.

[7] M. G. Gebreslassie, G. R. Tabor, M. R. Belmont, Numerical simulation of a new type of cross flow tidal turbine using OpenFOAM - Part I: Calibration of energy extraction, Renewable Energy 50 (2013) 994-1004. doi:10.1016/j.renene.2012.08.065.

[8] M. G. Gebreslassie, G. R. Tabor, M. R. Belmont, Numerical simulation of a new type of cross flow tidal turbine using OpenFOAM - Part II: Investigation of turbine-to-turbine interaction, Renewable Energy 50 (2013) 1005-1013. doi:10.1016/j.renene.2012.08.064.

[9] M. G. Gebreslassie, G. R. Tabor, M. R. Belmont, Investigation of the performance of a staggered configuration of tidal turbines using CFD, 
Renewable Energy 80 (2015) 690-698. doi:10.1016/j.renene.2015. 03.001.

[10] B. G. Sellar, D. R. J. Sutherland, MD 3.8 - Tidal energy site characterisation at the fall of warness, EMEC, UK, techreport, available from: http://redapt.eng.ed.ac.uk/ (2015).

[11] T. H. E. Clark, Turbulence in Marine Environments ( TiME ): A framework for understanding turbulence and its effects on tidal devices., In review.

[12] T. Stallard, R. Collings, T. Feng, J. Whelan, Interactions betweem tidal turbine wakes: Experimental study of a group of 3-bladed rotors, Philosophical Transactions of the Royal Society of London: A 371 (1985) (2013) 1471-2962.

[13] S. G. Parkinson, T. Stallard, M. Thomson, a. Wickham, R. Willden, Comparison of scale model wake data with an energy yield analysis tool for tidal turbine farms, 4th International Conference on Ocean Energy (2012) 2-6.

[14] T. Daly, L. Myers, A. Bahaj, Numerical analysis of the acceleration and wake effects resulting from changes in tidal turbine array position in a channel (2011) 1-9.

[15] T. Nishino, R. H. J. Willden, The efficiency of an array of tidal turbines partially blocking a wide channel, Journal of Fluid Mechanics 708 (2012) 596-606. doi:10.1017/jfm.2012.349. 
[16] S. Draper, T. Nishino, Centred and staggered arrangements of tidal turbines, Journal of Fluid Mechanics 739 (2014) 72-93. doi:10.1017/ jfm. 2013.593.

[17] S. Draper, T. Stallard, P. Stansby, S. Way, T. Adcock, Laboratory scale experiments and preliminary modelling to investigate basin scale tidal stream energy extraction, in: 10th European Wave and Tidal Energy Conf., Aalborg, Denmark, 2013.

[18] J. G. Leishman, Challenges in modelling the unsteady aerodynamics of wind turbines, Wind Energy 5 (2-3) (2002) 85-132. doi:10.1002/we. 62.

[19] R. Vennell, S. W. Funke, S. Draper, C. Stevens, T. Divett, Designing large arrays of tidal turbines: A synthesis and review, Renewable and Sustainable Energy Reviews 41 (2015) 454-472. doi:10.1016/j.rser. 2014.08 .022$.

[20] L. E. Myers, A. S. Bahaj, An experimental investigation simulating flow effects in first generation marine current energy converter arrays, Renewable Energy 37 (1) (2012) 28-36. doi:10.1016/j.renene.2011. 03.043.

[21] S. C. Cooke, R. H. J. Willden, B. W. Byrne, T. Stallard, A. Olczak, Experimental Investigation of Thrust and Power on a Partial Fence Array of Tidal Turbines, in: 11th European Wave and Tidal Energy Conference, Nantes, France, 2015, pp. 1-10. 
[22] P. Mycek, B. Gaurier, G. Germain, G. Pinon, E. Rivoalen, Numerical and experimental study of the interaction between two marine current turbines, International Journal of Marine Energy 1 (2013) 70-83. arXiv: 1310.4921, doi:10.1016/j.ijome.2013.05.007.

[23] A. Robinson, D. Ingram, I. Bryden, T. Bruce, The effect of inlet design on the flow within a combined waves and current flumes, test tank and basins, Coastal Engineering 95 (2015) 117-129. doi:10.1016/j. coastaleng. 2014.10.004.

[24] A. Robinson, I. Bryden, D. Ingram, T. Bruce, The use of conditioned axial flow impellers to generate a current in test tanks, Ocean Engineering 75 (2014) 37-45. doi:10.1016/j.oceaneng.2013.10.016.

[25] D. R. Noble, T. Davey, H. C. M. Smith, P. Kaklis, A. Robinson, T. Bruce, Spatial variation in currents generated in the FloWave Ocean Energy Research Facility, in: 11th European Wave and Tidal Energy Conference, Nantes, France, 2015.

[26] D. R. J. Sutherland, D. R. Noble, J. Steynor, T. Davey, T. Bruce, Characterisation of Current and Turbulence in the FloWave Ocean Energy Research Facility, Ocean Engineering 139, 15 July 2017, Pages (2016) $103-115$.

[27] J.-B. Richard, J. Thomson, B. Polagye, J. Bard, Method for identification of Doppler noise levels in turbulent flow measurements dedicated to tidal energy, International Journal of Marine Energy 3-4 (2013) 52-64. doi:10.1016/j.ijome.2013.11.005. 
[28] A. Chadwick, J. Morfett, M. Borthwick, Hydraulics in civil and environmental engineering, 4th Edition, 2004.

[29] S. C. M. Claudio A Consul, Richard H. J Willden, Blockage effects on the hydrodynamic performance of a marine cross-flow turbine, Philosophical Transactions of the Royal Society Adoi:10.1098/rsta.2012.0299.

[30] P. Myceka, B. Gaurierb, G. Germainb, G. Pinona, E. Rivoalena, Experimental study of the turbulence intensity effects on marine current turbines behaviour. Part I: One single turbine, Renewable Energy 66 (June) (2014) 729-746.

[31] A. M.-J. D. O. C. M. T. O. C. B. P. P. R. G. I. O. S. T. R. Poole, Non-dimensional scaling of tidal stream turbines, Energy.

[32] A. P. B. Michael S. Selig, James J. Guglielmo, P. Giguere, Summary of low-speed airfoil data vol. 1, Tech. rep., Department of Aeronautical and Astronautical Engineering, University of Illinois at Urbana-Champaign. SOARTECH Publications. (1995).

[33] P. G. L. M. A. Bahaj, Experimental and numerical results of rotor power and thrust of a tidal turbine operating at yaw and in waves, in: World Renewable Energy Congress - Marine and Ocean Technology, Linkoping, 2011.

[34] R. Malki, I. Masters, A. J. Williams, T. Croft, Planning tidal stream turbine array layouts using a coupled blade element momentum computational fluid dynamics model, Renewable Energy 63 (2014) 46-54. doi:10.1016/j.renene.2013.08.039. 
716

717

718

719

720

721

722

723

724

[35] A. S. Bahaj, A. F. Molland, J. R. Chaplin, W. M. J. Batten, Power and thrust measurements of marine current turbines under various hydrodynamic flow conditions in a cavitation tunnel and a towing tank, Renewable Energy 32 (3) (2007) 407-426. doi:10.1016/j.renene.2006.01. 012.

[36] S. H. Salter, J. R. M. Taylor, Vertical-axis tidal-current generators and the Pentland Firth, Proceedings of the Institution of Mechanical Engineers, Part A: Journal of Power and Energy 221 (2) (2007) 181-199. doi : 10.1243/09576509JPE295. 